Results The total economic cost of the intervention over 4 years for 23 districts was US\$27341121(range per district US\$307597-US $\$ 3147790$ ) for an estimated target population of 93345. In year 4, the average annual economic cost per estimated population at the NGO level across the 23 districts was US\$70 (US\$30-174). The average annual financial cost at current prices was US\$59. Over the four years, at the NGO level, capital costs accounted for $10 \%$ of total costs. The main recurrent costs were personnel costs (46\%) and the materials and supplies for sexually transmitted infections (STI) services (13\%). Examining both SLP and NGO costs, programme management, information and grant management costs activities accounted for $27 \%$ of total cost; followed by capacity building $(20 \%)$, STI services (19\%), peer outreach (including behaviour change communication, condom provision) (16\%) and structural interventions (11\%). The proportion of cost that was spent on direct services such as peer outreach and STI services increased as the programme scaled up. Costs for structural activities also increased from $5 \%$ in year 1 to $11 \%$ by year 4 .

Conclusions Assessing costs over the life of the project helps to identify how costs vary with the changing needs and strategies of the programme. We will conduct further analysis to examine which factors most influence costs (local price/wages, programme intensity, community involvement etc). This cost data can assist the realistic planning of large scale long term HIV prevention programmes in the future.

\section{P2-S5.08 KNOWLEDGE ABOUT STD TRANSMISSION AND CLINICAL CHARACTERISTICS AMONG CONSCRIPTS IN BRAZIL, 2007}

doi:10.1136/sextrans-2011-050108.350

${ }^{1} \mathrm{D}$ Ribeiro, ${ }^{1} \mathrm{~V}$ M Pinto, ${ }^{2} \mathrm{~A}$ Espinosa, ${ }^{1} \mathrm{E}$ Rezende, ${ }^{1} \mathrm{G}$ Pereira, ${ }^{3} \mathrm{M}$ Barbosa. ${ }^{1}$ Ministry of Health, Brasilia, Brazil; ${ }^{2}$ UFES, Brazil; ${ }^{3}$ Federal District Government, Brazil

Our goal was to describe knowledge of military conscripts related to STDs. A self-applied questionnaire with demographic characteristics and STD clinics; knowledge of transmission modes and preferred access to information about STDs was applied. Variables associated with outcome were: being 19-20 years [OR 1.2 (95\% CI 1.18 to 1.32)]; STD transmission by eating contaminated food [OR 2.2 (95\% CI 1.96 to 2.55)], for bathing in rivers / beaches [OR $1.5(95 \%$ CI 1.27 to 1.88 )]; by mosquitoes [OR 1.5 (95\% CI 1.38 to 1.65$)$ ], and for having sexual intercourse $\leq 14$ years [OR 1.4 (95\% CI 1.33 to $1.55)]$. The variables were negatively associated: being white [OR 0.9 (95\% CI 0.82 to 0.91$)$ ], namely the transmission by sharing syringes and needles [OR 0.7 (95\% CI 0.62 - to 0.78 )], by transmission from mother to child during childbirth and breastfeeding [OR 0.6 (95\% CI 0.58 to 0.69 )], using a condom at last sex [OR 0.8 (95\% CI 0.71 to 0.85$)$ ]; MSM [OR 0.7 (95\% CI 0.60 to 0.92 ) and know that sex without condom use increases the risk of transmission [OR 0, 4 (95\% CI 0.37 to 0.51)]. STD education programs must be dynamic and comprehensive, involving teens, their parents and educators, once isolated knowledge about modes of transmission of STD is not enough to help protect.

\section{P2-S5.09 TRANSGENDERS AND HIV: A LITERATURE REVIEW OF HIV RISK BEHAVIOURS AND PREVALENCE RATES}

doi:10.1136/sextrans-2011-050108.351

${ }^{1} \mathrm{~S}$ Butt, ${ }^{1} \mathrm{~L}$ Mena, ${ }^{2} \mathrm{C}$ Muzny. ${ }^{1}$ University of Mississippi Medical Center, Jackson, USA; ${ }^{2}$ University of Alabama, Birmingham, USA

Background Transgenders (TG) are a marginalised population that has been greatly impacted by the HIV/AIDS epidemic. The objective of this study is to review the current literature citing risk factors and prevalence rates for HIV infection among male to female (MTF) and female to male (FTM) TG in the USA.

Methods A review of the original research articles published from 1981 to 2010 was performed through a Pubmed search using the terms "HIV risk" and "Transgender".

Results Thirty-five articles were identified, with MTF described in thirty-three and FTM described in nine. Collectively all racial groups were represented. The majority of research was done in large urban cities in the Southwest, Midwest, and Northeast. Most studies relied on self-report of HIV infection; and only six studies tested participants for HIV. The prevalence rates of HIV infection ranged from $2.2 \%$ to $68.0 \%$. Unprotected receptive anal intercourse (URAI), commercial sex work (CSW), and African American (AA) race were the most frequent HIV risk factors identified. Homelessness and financial instability were frequent among adolescents. When compared to men who have sex with men (MSM) and heterosexual females, MTF are more likely to have URAI with multiple sexual partners. Among MTF participants, AA and Hispanics were more likely to participate in CSW than Caucasians. Self reported prevalence of HIV was low in FTM population (0-2\%), except in one study which tested for HIV and found similar HIV prevalence in both FTM (10\%) and MTF (10\%); risk factors associated with HIV infection in this study were unprotected sex with a partner of unknown HIV status and location in an urban metropolitan area. Conclusions HIV rates among TG are high, especially among AA and Hispanics. Majority of MTF participate in high risk sexual activities. Geographically and ethnically diverse studies including MTF and FTM are needed to test HIV prevalence and associated risk factors in TG population.

\section{P2-S5.10 SEXUAL BEHAVIOURS AMONG INJECTING DRUG USERS (IDUS) IN NORTH-EAST INDIA}

doi:10.1136/sextrans-2011-050108.352

\section{R K Mishra. Emmanuel Hospital Association, Guwahati, India}

Background The North-East Indian states of Nagaland and Manipur have among the highest HIV prevalence in India, fuelled by an epidemic of injecting drug use. In 2008, the HIV prevalence among IDUs in Manipur was 28.65\% and in Nagaland was 3.16\% in 2008 as per National Surveillance data. Proximity to Myanmar, high unemployment and ongoing violent insurgencies serve to exacerbate the problem. Project ORCHID, an initiative funded by the Bill and Melinda Gates Foundation, has been running HIV prevention programs with high risk groups through local NGOs in these two states since 2004. Methodology: To evaluate risk behaviours among the IDU communities served by Project ORCHID, polling booth surveys (PBS) were conducted in all 26 intervention sites across both states in 2010. Participants were purposively selected from a broad geographical area, and asked to anonymously answer questions through a ballot box.

Results In total, 558 IDUs in Manipur and 440 IDUs in Nagaland were sampled for the PBS. They reported high rates of access to needles/syringes from ORCHID projects (82\% in Manipur and 96\% in Nagaland). In both states, three-quarters had injected in the previous week. Among these, the mean weekly injection frequency was 11 times in Manipur and 5 in Nagaland. The proportion of injecting episodes using a clean needle/syringe was $77 \%$ in Manipur and $94 \%$ in Nagaland. Needle sharing in the previous month was reported by 38\% in Manipur and 25\% in Nagaland. Manipur had less IDUs who had been sexually active in the previous week $(46 \%$ vs $71 \%$ in Nagaland) but consistent condom use was lower in Manipur than Nagaland (30\% vs $76 \%$ ).

Conclusion Higher rates of risky injecting and sexual behaviour were found in Manipur IDUs, which together with the much higher 
injection frequency and HIV prevalence of $28.65 \%$ underlines the need for continued behaviour change communication. Low injecting rates and moderate to high sexual activity among Nagaland IDUs indicates communication strategies should shift towards sexual risk behaviours. The 3rd PBS is underway and will enable to see how behaviours are changing over time.

\section{Social and behavioural aspects of prevention poster session 6: Men who Have Sex with Men}

\section{P2-S6.01 SOCIAL, BEHAVIOURAL CHARACTERISTICS, AND SEROPREVALENCE OF HIV INFECTION AMONG MEN WHO HAVE SEX WITH MEN (MSM): ABOUT 92 CASES COLLECTED IN A COMMUNITY MEDICAL CENTER NAMED CENTRE OASIS “OF ASSOCIATION AFRICAN SOLIDARITÉ (AAS), OUAGADOUGOU, BURKINA FASO"}

doi:10.1136/sextrans-2011-050108.353

\begin{abstract}
${ }^{1}$ Ter Tiero Elias Dah, ${ }^{2}$ T S Koala. ${ }^{1}$ Association African Solidarité, Ouagadougou, Burkina
\end{abstract} Faso; ${ }^{2}$ GGlobal Fund, Burkina Faso, Ouagadougou, Burkina Faso

Background Like many African countries, the issue of sex between men in Burkina Faso remains taboo and is often the cause of social exclusion. Our objective in this study was to determine social and behavioural characteristics and seroprevalence of HIV infection among MSM in Ouagadougou.

Methods A questionnaire (closed questions) written by the medical team at Centre Oasis" was submitted to the MSM. Our sample of 92 consisted of MSM who frequented the center and some who did not. Data were collected by medical staff and peer educators. They were: -Social, -Behavioural, -The results of HIV serology.

Results The average age of MSM surveyed was 28.6 years with extremes of 18 and 56 years. MSM were predominantly students with a proportion of $34.8 \%$. $21.7 \%$ were traders, $2.2 \%$ were unemployed. More than half of respondents were single (54.8\%). $22.8 \%$ were living with a common-law wife, and $20.7 \%$ were legally married. $78.3 \%$ of MSM had had sex with a woman. 2 / 3 of all MSM (65.2\%) were bisexual. In $90.2 \%$ of cases, the MSM used condoms two out of three times during sexual intercourse $0.77 .2 \%$ of MSM used water-based gels two out of three times during sexual intercourse. The majority of MSM $(82,6 \%)$ had more than one sexual partner. In $82.1 \%$, none of the MSM had discussed their situation with their family for fear of being rejected. Of 55 MSM who had had an HIV test, 8 had been diagnosed positive for HIV giving a prevalence of $14.5 \%$.

Conclusion In our study, it appears that the MSM community in Ouagadougou is highly vulnerable given the high rate of HIV seroprevalence and its marginalisation. Targeted interventions for the recognition ofsupport are needed to help sustain the achievements of the national fight against HIV.

\section{P2-S6.02 USE OF A COMPUTER ALERT INCREASES DETECTION OF EARLY, ASYMPTOMATIC SYPHILIS AMONG HIGHER RISK MEN WHO HAVE SEX WITH MEN}

doi:10.1136/sextrans-2011-050108.354

${ }^{1} \mathrm{M}$ Bissessor, ${ }^{1} \mathrm{C}$ Fairley, ${ }^{2} \mathrm{D}$ Leslie, ${ }^{1} \mathrm{M}$ Chen. ${ }^{1}$ Melbourne Sexual Health centre, Carlton, Australia; ${ }^{2}$ VIDRL, Aruba

Background More frequent screening of higher risk men who have sex with men (MSM) for syphilis could reduce the transmission and prevalence of syphilis. This study assessed the impact of a computer alert on the rate of syphilis testing and diagnoses among higher risk MSM.

Methods In October 2008, a computer alert was introduced at the Melbourne Sexual Health Centre. This alert appeared during consultations for MSM who reported more than 10 male partners in the prior 12 months, reminding clinicians to test such higher risk men 3 monthly for syphilis. Syphilis testing rates and diagnoses among MSM were determined for the 12 months before and the 12 months after the introduction of the alert.

Results The proportion of MSM who were identified as being higher risk who were tested for syphilis in the two time periods increased from $77 \%(1559 / 2017)$ to $89 \%(1282 / 1445) \quad(p<0.001)$. The proportion of higher risk men diagnosed with early syphilis and who were asymptomatic for syphilis was $16 \%(5 / 31)$ and $53 \%(31 / 58)$ respectively $(p=0.001)$. By contrast, there was no significant increase in the proportion of MSM who were identified as being lower risk who were tested for syphilis: 65\% (1228/1885) and 68\% $(1667 / 2448)(p=0.4)$. Nor was there a significant increase in the proportion of lower risk men diagnosed with early syphilis who were asymptomatic: $10 \%(1 / 10)$ and $19 \%(3 / 16)$ respectively $(\mathrm{p}=0.6)$.

Conclusion The use of a computer alert was associated with increased syphilis testing of higher risk MSM attending a clinical service as well as increased detection of early, asymptomatic syphilis.

\section{P2-S6.03 ABSTRACT WITHDRAWN}

\section{P2-S6.04 NEEDS ASSESSMENT ON STI PREVENTION SERVICES AMONG MEN WHO HAVE SEX WITH MEN WITH HIV IN GUATEMALA CITY, 2010}

doi:10.1136/sextrans-2011-050108.356

${ }^{1} \mathrm{~N}$ Farach, ${ }^{1} \mathrm{~S}$ Boyce, ${ }^{2} \mathrm{C}$ Barrington, ${ }^{3} \mathrm{C}$ Galindo, ${ }^{1} \mathrm{G}$ Paz-Bailey. ${ }^{1} T E P H I N E T$, Tegucigalpa, Honduras; ${ }^{2}$ University of North Carolina, Chapel Hill, USA; ${ }^{3}$ Universidad del Valle, Guatemala City, Guatemala

Background STI prevention and support services for MSM living with HIV in Guatemala are limited. The aim of this study was to collect information on STI risk among MSM living with HIV to inform the development of integrated prevention and care services for this population.

Methods As part of a qualitative study with MSM in Guatemala City, nine MSM living with HIV were purposely selected and interviewed in March 2010. Interviews addressed STI-related risk behaviours, experiences with HIV/STI services, social network characteristics and life after HIV diagnosis. Participants were recruited through referral from NGO representatives, key informants, clinic staff and other participants. In-depth interviews were recorded and transcribed and field notes were incorporated into the transcriptions. After multiple readings of the transcripts, key themes were identified and a codebook was developed. Codes were applied using the qualitative software Atlas.ti. Data was further analysed using analytic memos and discussions with research team.

Results Despite reporting HIV-related risk behaviours, including multiple, concurrent sexual partners, inconsistent condom use, and alcohol and drug use, most participants did not report an STI infection after their HIV diagnosis. Attitudes towards current HIV/AIDS treatment services tended to be favourable, especially among those who compared them to earlier treatment experiences, and participants described preferences for a one-stop venue for sexual health services. Participants were generally not familiar with STI services and identified several barriers to access including insufficient information on STI, lack of confidentiality and fear 